\title{
Abnehmen ohne Diät - Illusion oder Möglichkeit?
}

\author{
Eine Entgegnung zur Vorstellung der Daten zu Refigura (BEFORE-Studie, \\ Z Phytother 2018; 39(4): 159-164)
}

Hans Konrad Biesalski

Universität Hohenheim

Abends vor dem Fernseher eine Tüte Chips verzehren, am Nachmittag von Zeit zu Zeit ein Stück Torte und zwischen den Mahlzeiten immer wieder mal was Süßes. Keine Einschränkungen beim Essen, kein lästiges Kalorienzählen und auch keine überflüssige Bewegung. Dem Belohnungssystem, das uns immer wieder zum Naschen anregt freien Raum lassen, einfach zu jeder Mahlzeit eine Pille einwerfen, die alles was dick macht entfernt und vielleicht sogar noch überflüssige Pfunde abbaut. Abnehmen ohne Diät, das verspricht jetzt eine breit gestreute Verbraucherwerbung aufgrund einer in dieser Zeitschrift veröffentlichten Studie zur Wirkung von Refigura [1].

Refigura ist ein Medizinprodukt, das nicht dem Arzneimittelgesetz, sondern dem Medizinproduktegesetz unterliegt. In diesem sind die Vorgaben der Veröffentlichung einer klinischen Studie noch nicht vorgeschrieben. Man vertraute in der EU auf die freiwilligen Vereinbarungen wie die Deklaration von Helsinki, CONSORT-Statement usw. Diese Lage wird nun ausgenutzt, indem sich Studien das Mäntelchen der Wissenschaftlichkeit umhängen und so dem Verbraucher das Gefühl vermitteln, dass er es mit wissenschaftlich gesicherten Ergebnissen zu tun hat, die aufgrund der nicht eingehaltenen Mindeststandards das vermittelte Resultat fragwürdig erscheinen lassen.

Die in der Zeitschrift für Phytotherapie veröffentlichte „BEFORE-Studie“ zur Gewichtsreduktion mit Refigura ohne Änderung der Lebens- und Essgewohnheiten ist ein Beispiel für eine solche Studie. Als Prüfmedikation wurden drei unterschiedlich wirkende Substanzen gemeinsam eingesetzt, das aus dem Schimmelpilz Aspergillus niger gewonnene Chitosan mit einem Anteil von ca. $15 \%$ $\beta$-Glucan [2] und Glucomannan.

Chitosan soll mit Fetten eine Emulsion im Gastrointestinaltrakt bilden, was dazu führt, dass ein Teil der Fette nicht absorbiert wird. Gleichzeitig steigt die Magenfüllung, was über hormonelle Effekte (Ghrelin) zu einer gewissen Sättigung beitragen kann. Glucomannan ist ein
Quellstoff, der wie viele unverdauliche Ballaststoffe in geringem Maße Fette und Gallensäuren adsorbiert und so der Verdauung entzieht. $\beta$-Glucan ist ein natürlicher Bestandteil der Zellwände von Pilzen und Pflanzen, wird durch menschliche Enzyme nicht verdaut, aber stattdessen im Dünndarm aufgenommen und stimuliert das mucosale und das systemische Immunsystem [3], [4]. Die Autoren wollen nun durch Kombination der drei Substanzen prüfen, inwieweit eine Gewichtsreduktion ohne Änderung des Lebens- und Ernährungsstils erreicht werden kann.

Dazu erhalten die Teilnehmer ( $\mathrm{n}=165$ mit BMI zwischen 27 und 35; 81,2\% weiblich), randomisiert in drei Gruppen (zwei Verum-Applikationen in unterschiedlicher Konzentration [Chitosan inklusive $\beta$-Glucan/Glucomannan; Verum 1: 1,5/3,0 g bzw. Verum 2: 3,0/4,02 g] und einer Placebogruppe) die Prüfmedikation über einen Zeitraum von 56 Tagen. Primärziel ist das Körpergewicht, Sekundärziele sind Veränderung des Bauchumfangs, des Körperfettanteils und der Lebensqualität.

\section{Primärziel der BEFORE-Studie: Gewichtsverlust}

Die Autoren kommen zu dem Ergebnis, dass die Anwendung der Prüfmedikation zu einem Gewichtsverlust von $0,89 \mathrm{~kg}$ gegenüber Placebo geführt hat. Dieser sei signifikant. Dies ist aber schwer nachvollziehbar, wenn sich das mittlere Gewicht in 8 Wochen nur von 92,0 auf 91,11 kg reduziert.

Die Auswertung der Studie lässt eine Reihe von Fragen offen, wie z.B. die der Compliance. Diese war bereits gegeben, wenn die Probanden nicht weniger als $30 \%$ der verordneten Menge des Präparates eingenommen hatten. In seriösen klinischen Studien ist die Grenze bei $80 \%$. In ihrem Fazit kommen die Autoren zu dem Ergebnis, dass die Kombination übergewichtigen Patienten beim Abnehmen helfen kann, weil damit auch ohne Umstellung der Lebens- oder Essgewohnheiten eine signifikante 
Gewichtsreduktion herbeigeführt wird, die, so die Autoren, bei der höheren Dosierung entsprechend höher ausfällt. „Höher“ sind in diesem Fall $100 \mathrm{~g}$ in 8 Wochen! Entsprechend internationaler Empfehlungen sollte der Maßstab für eine erfolgreiche Gewichtsreduktion sein, $5 \%$ Körpergewicht (hier 4,64 kg im Patientenkollektiv) in Schritten von ca. $500 \mathrm{~g} /$ Woche abzunehmen [5].

Als statistische Analyseverfahren beschreiben die Autoren die Verwendung des sogenannten Full Analysis Set (FAS), dem alle 165 randomisierten Probanden angehören würden, die das Prüfprodukt mindestens einmal erhalten hatten. Außerdem das Per Protocoll Analysis Set (PPS), in dem alle Probanden erfasst werden, die bis zum Ende der Studie ohne Prüfplanverletzung dabei waren. Bei PPS wurden 110 Probanden ausgewertet, d.h. 55 Probanden wurden somit nicht in die Auswertung einbezogen. Die FAS-Ergebnisse werden aber nicht dargestellt, obwohl dies relevant wäre. Nur im FAS kann eine Analyse der Auswirkung der Prüfplanverletzungen in Bezug auf das Ergebnis erfolgen. Die Beurteilung der Wirkung nach PPS alleine ist mehr als fragwürdig, da damit nicht der geplante Durchschnitt dargestellt ist, sondern der positive 2 / 3-Anteil der Studienteilnehmer.

In einer früheren Studie mit Kombination der beiden Substanzen (je 1,2 g Chitosan und Glucomannan an 22 Probanden über einen Zeitraum von 28 Tagen) konnte eine signifikante Senkung des Cholesterins (HDL, LDL, Gesamt) beobachtet werden [6]. Die Ausscheidung von Cholesterin und Gallensäuren nahm zu, die Fettausscheidung änderte sich nicht. Ein Effekt auf das Körpergewicht blieb jedoch aus. Dies kann an der im Vergleich zu der Verum-1-Gruppe niedrigeren Menge an Glucomannan, aber auch am kürzeren Beobachtungszeitraum gelegen haben. Höhere Dosierungen von Glucomannan $(3 \times 1,33 \mathrm{mg} /$ Tag für 8 Wochen) führen zu einer Senkung des LDL- und Gesamtcholesterins bei Probanden (23 Verum, 24 Kontrolle), die ihre Ernährung und körperliche Aktivität nicht verändert hatten, was mit aufwendiger Prüfung durch Fragebögen sichergestellt wurde [7]. Ein Effekt auf Körperfett oder Körpergewicht wurde dagegen nicht beobachtet.

\section{Sekundärziele: Anthropometrische und Ernährungsdaten der Teilnehmer}

Die wesentliche Aussage, dass der Gewichtsverlust ohne Änderung der Ernährung und Lebensgewohnheiten erfolgt ist, ist an keiner Stelle der Studie belegt. So wird zwar erklärt, dass die Teilnehmer alle 14 Tage einbestellt wurden und dabei der Ernährungsstatus einschließlich Körperfettanteil erhoben wurde, die aufbereiteten Daten werden dem Leser allerdings vorenthalten.

Die Analyse des Körperfettanteils kann nur durch die sogenannte Body Impedanz Analyse (BIA) erfolgen. Dies ist wichtig, wenn eine Beurteilung des BMl erfolgt, da dieser nicht unbedingt nur durch die Fettmasse, sondern auch die fettfreie Körpermasse erklärt wird. Das ist besonders dann unerlässlich, wenn die Auswertung sich am BMI orientiert, wie in der Studie geschehen. BIA-Messungen sind, besonders dann, wenn sie im Rahmen einer klinischen Studie eingesetzt werden, sehr zeitaufwendig (Wiederholungsmessung) und brauchen gut geschulte Kräfte. Es ist schlicht nicht vorstellbar, dass alle 14 Tage 165 Teilnehmer auf diese Weise untersucht wurden. Daher wundert es auch nicht, wenn die aufbereiteten Daten in der Arbeit nicht weiter angegeben werden.

Die Erfassung der Ernährungsweise muss nach standardisierten Verfahren durch entsprechend geschulte Interviewer erfolgen (z. B. 3-day food record oder $24 \mathrm{~h}$ recall). In der Studie selbst wird lediglich angegeben, dass die Studienschwestern nach der Ernährung gefragt haben. Inwieweit die Teilnehmer spezielle Ernährungsformen hatten, die z. B. reich an Kohlenhydraten oder Ballaststoffen waren, oder Low-fat-Produkte bevorzugt wurden, bleibt ebenso offen, wie Unterschiede in Energieaufnahme und -verbrauch. Eine Studie, die als Indikator Ernährungsverhalten hat-und dies ist ein wesentlicher Aspekt dieser Studie -, kann das individuelle Ernährungsverhalten nicht nebenbei als geprüft erwähnen, ohne Daten vorzulegen. Gleiches gilt für die körperliche Aktivität: Auch hier sind internationale Standards festgelegt, wie das International Physical Questionnaire [8].

\section{Unerwünschte Nebenwirkungen}

$56 \%$ der Nebenwirkungen fielen auf Erkrankungen des Gastrointestinaltraktes. Was das bedeutet und in wie weit dies auch zu Drop out geführt hat, bleibt offen. Im Sinne des Verbraucherschutzes wäre es sicherlich gut gewesen, wenn Hinweise auf mögliche weitere Nebenwirkungen, wie der Einfluss auf die Vitaminversorgung, erfolgt wären.

\section{Vitamine}

Eine Einnahme des Präparates (verfügbar in der Verum1-Dosis) über längere Zeit birgt Risiken, die aus verschiedenen Studien bekannt sind und dem Konsumenten mitgeteilt werden sollten.

Bei Pflanzensterolen, die die Absorption von Cholesterin kompetitiv hemmen, sind Effekte auf die Absorption von fettlöslichen Vitaminen ( $E, A, D)$ sowie auf fettlösliche Carotinoide vielfach untersucht worden. Bei der Beurteilung der Bioverfügbarkeit sind streng genommen nur die Carotinoide brauchbar, da durch die homöostatische Regulierung des Vitamin A und die Vitamin-D-Synthese der Haut Schwankungen durch Unterschiede in der Bioverfügbarkeit kaum zu erkennen sind. Das bestätigt sich in einer ersten Metaanalyse (18 Studien) [9], bei der eine 
signifikante Reduktion von Vitamin E, $\alpha$-, $\beta$-Carotin und Lycopin, nicht aber von Retinol, und Vitamin D nach Verzehr von Pflanzensterolen und -stanolen bestand. Dies kann auch durch die Reduktion des zirkulierenden Cholesterins erklärt werden, da Vitamin E und andere Carotinoide (Ausnahme $\beta$-Carotin) in Lipoproteinen transportiert werden. In einer weiteren kürzlich erschienenen Metaanalyse [10], die 52 Studien erfasst, findet sich eine moderate Reduktion des Vitamin E (10\% median) und eine signifikante Verringerung des $\beta$-Carotins um $24 \%$. Als Ursache für die verringerten Blutspiegel bei Verzehr von Phytosterolen werden Interaktionen mit der micellaren Absorption oder dem Einbau in die Lipoproteine diskutiert [11].

Im Unterschied zu den Phytosterolen wirken Chitosan und Glucomannan wohl nicht selektiv auf die Cholesterin-Absorption, sondern auf die gesamte Absorption von Fetten und fettlöslichen Verbindungen. Im Gegensatz zu den Phytosterolen ist der Einfluss auf die Absorption fettlöslicher Vitamine und Carotinoide bisher nur in wenigen Studien geprüft. Jede der beiden Substanzen führt nicht nur zu einer erwünschten Reduktion der Bioverfügbarkeit von Fetten, sondern auch von fettlöslichen Vitaminen und Mineralen [12], [13]. Was das für die in der BEFORE-Studie gewählte Kombination bedeutet, kann nur schwer gesagt werden, der Verbraucher sollte jedoch darauf hingewiesen werden.

Wie es mit der Bioverfügbarkeit essenzieller fettlöslicher Verbindungen bei Anwendung der Kombination aussieht, ist ebenso wenig geklärt wie die Frage, inwieweit die dem enterohepatischen Kreislauf unterliegenden Mikronährstoffe davon betroffen sind. Auch für die Wirkungen des $\beta$-Glucan im vorliegenden Zusammenhang sind keine Daten auffindbar. Um eine solche Kombination als sicher für den Verbraucher zu empfehlen, sollten solche Daten bekannt sein.

Wenn die Autoren dem Konsumenten suggerieren, dass er seinen Ernährungsstil nicht ändern, sondern nur das kombinierte Präparat lange genug einnehmen muss, bis er genügend Gewicht verloren hat ( $890 \mathrm{~g}$ in 8 Wochen), so wird dabei übersehen, dass der Konsument ein nicht unerhebliches Risiko hinsichtlich der Versorgung mit fettlöslichen Mikronährstoffen, aber auch Mineralien eingeht. Vor diesem Hintergrund ist eine Bewerbung eines solchen Präparates aus gesundheitlicher Sicht höchst bedenklich. Nicht umsonst wird immer wieder darauf hingewiesen, dass die Mikronährstoffversorgung bei Adipösen im Rahmen vieler Untersuchungen als kritisch angesehen werden muss [14].

Dem Verbraucher zu suggerieren, er könne so weiterleben wie bisher und möglicherweise ungesunde Ernährungs- und Lebensgewohnheiten beibehalten, steht im Widerspruch zu den Empfehlungen der WHO und aller seriöser internationaler Fachgesellschaften, vgl. z. B. interdisziplinäre S3-Leitlinie zur Prävention und Therapie der Adipositas der Deutschen AdipositasGesellschaft und der Deutschen Diabetes Gesellschaft [15]. Übergewichtige sollten bestärkt werden, ihr Ernährungsverhalten zu ändern und die tägliche physische Aktivität zu erhöhen mit dem Ziel, gesund abzunehmen.

Der Ansatz, dieses „Wundermittel“ verhelfe ohne Umdenken und ohne Änderung der Lebensgewohnheiten zu einer schönen Figur, ist grundsätzlich falsch und schadet letztendlich der Gesundheit.

\section{Fazit}

Die BEFORE-Studie will einen Gewichtsverlust durch Anwendung eines Kombinationspräparates zeigen. Die methodischen Schwächen, die unzulänglich verfügbaren Daten und die ungeklärten Fragen in der Auswertung lassen das Ergebnis mehr als fragwürdig erscheinen. Gerade in der Phytomedizin sollte auf die Seriosität von Studien in Planung, Durchführung und Veröffentlichung geachtet werden, um nicht zu einer Diskreditierung dieser wichtigen Wissenschaften beizutragen. Dazu gehört, dass klinische Studien hinsichtlich der international anerkannten Vorgehensweise gemäß CONSORT-Statement [16] durchgeführt wurden. Diese wurden in der BEFORE-Studie grob vernachlässigt. So fehlen die Einschluss- und Ausschlusskriterien bzw. werden nur teilweise angegeben, die Biometrie ist unzureichend (Mindestfallzahl liegt in der Verum-1-Gruppe über der tatsächlichen Fallzahl), die statistischen Methoden wurden nicht ausreichend durchgeführt (FAS), sekundäre Endpunkte nicht eindeutig beschrieben, Ausschlüsse werden nicht begründet. Die Diskussion wird nur marginal geführt und setzt sich nicht mit der aktuellen Literatur auseinander.

Zusammengenommen kann die Aussage zur Wirksamkeit der Kombination durch die veröffentlichten Studiendaten nicht nachvollziehbar belegt werden.

\section{Interessenkonflikt}

Der Autor war für die Firmen Ratiopharm, Pfizer, Certmedica und Herbalife Nutrition Institute als Consultant tätig.

Korrespondenzadresse

Prof. em. Dr. med. Hans Konrad Biesalski
Universität Hohenheim
Garbenstr. 30
70593 Stuttgart
E-Mail: biesal@uni-hohenheim.de

Universität Hohenheim

70593 Stuttgart

E-Mail: biesal@uni-hohenheim.de 


\section{Literatur}

[1] Sokolowski T, Voet B. BEFORE-Studie. Nachweis der Wirksamkeit einer neuen, natürlichen Polyglucosamin / GlucomannanZubereitung zur Gewichtsreduktion ohne Änderung der Lebens- und Essgewohnheiten. Z Phytother 2018; 39: 159164

[2] Durchführungsverordnung (EU) 2017/2470 der Kommission vom 20.12.2017 zur Erstellung der Unionsliste der neuartigen Lebensmittel gemäß der Verordnung (EU) 2015/2283 des EU Parlaments und des Rates über neuartige Lebensmittel. https: / eur-lex.europa.eu/legal content / DE/TXT/?uri = uriserv:OJ.L_.201 7.351.01.0072.01.DEU\&toc $=$ OJ:L:2017:351:TOC

[3] Vos AP, M'Rabet L, Stahl B et al. Immune-modulatory effects and potential working mechanisms of orally applied nondigestible carbohydrates. Crit Rev Immunol 2007; 27: 97-140

[4] Batbayar S, Lee DH, Kim HW. Immunomodulation of fungal b-glucan in host defense signaling by dectin-1. Biomol Ther 2012; 20: 433-445

[5] Yumuk V, Tsigos C, Fried M et al.; Obesity Management Task Force of the European Association for the Study of Obesity. European guidelines for obesity management in adults. Obes Facts 2015; 8: 402-424

[6] Gallaher DD, Gallaher CM, Mahrt G] et al. A glucomannan and chitosan fiber supplement decreases plasma cholesterol and increases cholesterol excretion in overweight normocholesterolemic humans. J Am Coll Nutr 2002; 21: 428-433

[7] Keithley JK, Swanson B, Mikolaitis SL et al. Safety and efficacy of glucomannan for weight loss in overweight and moderately obese adults. J Obes 2013; 2013: 610908. doi:10.1155/ 2013/610908

[8] International Physical Activity Questionnaire - long last 7 days self-administered format. www.sdp.univ.fvg.it/sites/default/ files / IPAQ_English_self-admin_long.pdf
[9] Katan MB, Grundy SM, Jones P et al.; Stresa Workshop Participants. Efficacy and safety of plant stanols and sterols in the management of blood cholesterol levels. Mayo Clin Proc 2003; 78: 965-978. doi:10.4065/78.8.965

[10] Fardet A, Morise A, Kalonji E et al. Influence of phytosterol and phytostanol food supplementation on plasma liposoluble vitamins and provitamin A carotenoid levels in humans: An updated review of the evidence. Crit Rev Food Sci Nutr 2017; 57: 1906-1921

[11] Ntanios FY, Duchateau GS. A healthy diet rich in carotenoids is effective in maintaining normal blood carotenoid levels during the daily use of plant sterol-enriched spreads. Int J Vitam Nutr Res 2002; 72: 32-39

[12] Doi K, Matsuura M, Kawara A et al. Influence of dietary fiber (konjac mannan) on absorption of vitamin B12 and vitamin $\mathrm{E}$. Tohoku J Exp Med 1983; 141 (Suppl.): 677-681

[13] Deuchi K, Kanauchi O, Shizukuishi M, Kobayashi E. Continuous and massive intake of chitosan affects mineral and fat-soluble vitamin status in rats fed on a high-fat diet. Biosci Biotechnol Biochem 1995; 59: 1211-1216

[14] Biesalski HK. Vitamine und Minerale. Stuttgart: Thieme; 2016

[15] Deutsche Adipositas-Gesellschaft [u. a.]. Interdisziplinäre Leitlinie der Qualität S3 zur „Prävention und Therapie der Adipositas“. Version 2.0 (April 2014). https://www.adipo sitas-gesellschaft.de/fileadmin/PDF/Leitlinien/050 001I_S3_ Adipositas_Praevention_Therapie_2014-11.pdf

[16] CONSORT. www.consort-statement.org

Bibliografie

DOI https://doi.org/10.1055/a-0814-0616

Zeitschrift für Phytotherapie 2019; 40: 19-22

(c) Georg Thieme Verlag KG Stuttgart · New York ISSN 0722-348X 